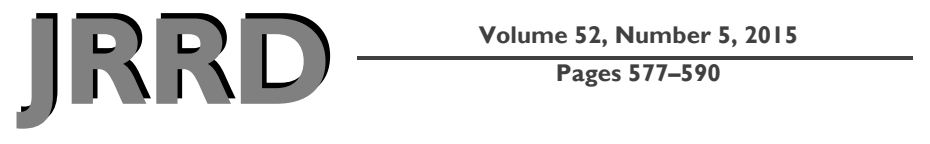

\section{Foot placement control and gait instability among people with stroke}

\author{
Jesse C. Dean, PhD; ${ }^{*}$ Steven A. Kautz, PhD \\ Ralph H. Johnson Department of Veterans Affairs Medical Center, Charleston, SC; and Division of Physical Therapy \\ and Department of Health Sciences and Research, College of Health Professions, Medical University of South Caro- \\ lina, Charleston, SC
}

\begin{abstract}
Gait instability is a common problem following stroke, as evidenced by increases in fall risk and fear of falling. However, the mechanism underlying gait instability is currently unclear. We recently found that young, healthy humans use a consistent gait stabilization strategy of actively controlling their mediolateral foot placement based on the concurrent mechanical state of the stance limb. In the present work, we tested whether people with stroke $(n=16)$ and age-matched controls $(n=19)$ used this neuromechanical strategy. Specifically, we used multiple linear regressions to test whether (1) swing phase gluteus medius (GM) activity was influenced by the simultaneous state of the stance limb and (2) mediolateral foot placement location was influenced by swing phase GM activity and the mechanical state of the swing limb at the start of the step. We found that both age-matched controls and people with stroke classified as having a low fall risk (Dynamic Gait Index [DGI] score >19) essentially used the stabilization strategy previously described in young controls. In contrast, this strategy was disrupted for people with stroke classified as higher fall risk (DGI </=19), particularly for steps taken with the paretic limb. These results suggest that a reduced ability to appropriately control foot placement may contribute to poststroke instability.
\end{abstract}

Key words: balance, biomechanics, foot placement, frontal plane, kinematics, motor control, muscle activity, stability, stroke, walking.

\section{INTRODUCTION}

Decreased gait stability, defined for the present work as a reduced ability to safely and confidently meet the mechanical demands of bipedal walking, can limit functional mobility following a stroke. People with stroke are more likely to experience a fall than uninjured individuals of the same age [1]. Among people with stroke classified as community ambulators, these falls often occur during walking [2-5]. Many falls within the poststroke population are not the result of environmental factors such as contact with an obstacle but are instead caused by internal factors related to balance abilities [3]. Mobility following a stroke can also be limited by an increased fear of falling [6]. Even in the absence of an injurious fall, fear of falling can be present [2], potentially decreasing activity participation and leading to eventual decreases in physical capacity [6-7].

Due to the clear importance of decreased stability among people with stroke, several clinical measures have been used to quantify balance performance or perception in this population. The Berg Balance Scale (BBS) is likely the most common clinical measure used to assess poststroke balance performance [8]. While this tool does not evaluate stability during walking, it has been used to

\footnotetext{
Abbreviations: $\mathrm{ABC}=$ Activities-Specific Balance Confidence scale, ANOVA = analysis of variance, BBS = Berg Balance Scale, CoM = center of mass, DGI = Dynamic Gait Index, EMG = electromyography, FP = foot placement, GM = gluteus medius, VA = Department of Veterans Affairs.

*Address all correspondence to Jesse C. Dean, PhD; 77 President St, MSC700, Charleston, SC 29425; 843-7929566; fax: 843-792-1358. Email: deaje@musc.edu http://dx.doi.org/10.1682/JRRD.2014.09.0207
} 
predict fall risk among people with stroke with mixed success [4,9-10]. The Dynamic Gait Index (DGI) was developed explicitly to test stability in various gait tasks [11] and has been validated in people with stroke [12]. Fear of falling among people with stroke has been quantified using the Activities-Specific Balance Confidence scale (ABC) and is associated with functional mobility beyond the effects of balance ability alone [13].

In addition to clinical measures of function, several laboratory-based measures have been developed with the goal of predicting an individual's fall risk. Kinematic variability during gait has been used as an indicator of stability, with increased stride time variability predicting increased fall risk in older adults [14] and lower DGI scores in people with stroke [15]. The patterns of wholebody angular momentum in the frontal plane may provide insight into gait instability, with fluctuations in this measure during single limb stance on the paretic side associated with BBS and DGI scores among people with stroke [16]. More complex indicators of gait stability, including Lyapunov exponents, Floquet multipliers, and long-term correlations, have also been associated with fall risk in an older population [17-19]. However, the ability of these metrics to predict fall risk among individuals with stroke has not yet been tested.

While both clinical and laboratory-based measures may allow predictions of which patients are at an increased risk for falls, they do not provide insight into the underlying mechanism of dysfunction. Such measures do not reveal which elements of the typical gait stabilization strategy have been disrupted and do not necessarily provide clinicians with information that would allow them to select the most effective focus for intervention. For example, a DGI score of $\leq 19$ may suggest that a patient is at an increased risk for falls [20]. However, this score does not indicate whether the underlying cause of the decreased stability is reduced strength, disrupted sensory feedback, an inability to appropriately integrate sensory information, or some other factor. Similarly, stride time variability could be measured and classified as higher than typical, but this information would not indicate the cause of the increased variability.

An alternative approach is to determine whether the typical gait stabilization strategies are disrupted following a stroke. To do so, we must first understand the control strategies that allow healthy humans to walk with a minimal fall risk and fear of falling. We recently identified a neuromechanical stabilization strategy that young, uninjured humans use to control mediolateral foot placement (FP) during both normal and mechanically perturbed walking [21]. Briefly, the mechanical state of the stance limb predictably influences simultaneous swing phase hip abductor activity in the contralateral limb; swing phase activity is higher when the center of mass $(\mathrm{CoM})$ is displaced farther mediolaterally from the stance foot. Increases in swing phase hip abductor activity cause the swing foot to be placed more laterally, a relationship modulated by the mechanical state of the lower limb at the start of the swing phase. These results indicate that uninjured humans meet the mechanical demands of bipedal walking by actively controlling mediolateral FP based on their perception of the mechanical state of the stance limb [21]. Therefore, the typical stabilization strategy could potentially be disrupted by poor sensation, a reduced ability to integrate sensory information, or an inability to appropriately recruit the hip abductors during swing.

The purpose of the present study was to determine whether the previously observed mediolateral stabilization strategy is disrupted in individuals who have experienced a stroke. We investigated unperturbed walking in people with stroke with a low fall risk (DGI $>19$ ), people with stroke with a higher fall risk (DGI $\leq 19$ ), and agematched uninjured controls. In each of these populations, we quantified the relationship between the mechanical state of the stance limb and the simultaneous swing phase hip abductor activity. We also quantified the relationship between swing phase hip abductor activity and subsequent mediolateral FP. We anticipate that these results will be an early step in identifying a mechanism underlying reduced gait stability in some individuals who experience a stroke.

We hypothesized that the typical neuromechanical stabilization strategy would be disrupted in participants with stroke. Also, we hypothesized that a more severe disruption would be present in participants at potentially greater risk of falling, as measured by the DGI. Such results would provide clear motivation to identify the specific sensory sources and sensorimotor integration processes that humans use to control FP.

\section{METHODS}

\section{Experimental Population}

The present study involved people with chronic stroke and age-matched control participants. Hemiparetic 
participants conformed to the following inclusion criteria: hemiparesis secondary to a unilateral stroke; absence of significant lower-limb joint pain, limb contractures, or major sensory deficits; ability to independently walk $>10 \mathrm{~m}$ on a level surface with an assistive device; and daily walking in the home. Potential participants were excluded if they had orthopedic or additional neurologic conditions beyond stroke or cardiovascular impairments contraindicative to walking. A total of 16 people with stroke and 19 age-matched controls participated in the experiments described.

\section{Experimental Protocol}

All participants performed overground walking trials in order to identify their self-selected overground walking speed and fastest-comfortable overground walking speed. Participants walked over a $14 \mathrm{ft}$ instrumented mat (GAITRite; Sparta, New Jersey) used to quantify walking speed. For the two self-selected trials, participants were instructed to walk at their normal speed. For the two fastest-comfortable trials, participants were instructed to walk at the fastest speed at which they felt safe. The selfselected and fastest-comfortable speeds were calculated as the averages across these two trials for each condition.

Participants with stroke also walked on an instrumented treadmill (Tecmachine; Andezieux-Boutheon, France) at their self-selected and fastest-comfortable speeds for a series of trials. For treadmill trials, selfselected speed was identified by changing the treadmill speed until participants reported being comfortable. The fastest-comfortable speed was identified by increasing the treadmill speed until participants reported being uncomfortable walking any faster. In order to record comparable control data for a range of speeds, control participants walked on the treadmill at their self-selected speed, fastest-comfortable speed, and over a range of previously determined speeds $(0.3,0.6,0.9,1.2,1.5$, and $1.8 \mathrm{~m} / \mathrm{s})$. All trials lasted $30 \mathrm{~s}$, and participants were provided with at least 1 min rest period between trials. During all treadmill walking trials, participants wore a harness attached to an overhead rail. This harness did not support body weight but would have prevented a fall in case of a loss of balance.

Because walking speed may influence stabilization strategy [22] and mediolateral FP location [23], we restricted our analysis to trials within a relatively narrow range of speeds. For participants with stroke, we analyzed only treadmill trials in which participants walked at speeds of $0.8 \mathrm{~m} / \mathrm{s}$ or higher (in at least one of the selfselected and fastest-comfortable trials), a value that has previously been related to the ability to effectively ambulate in the community [24]. For control participants, we analyzed only treadmill trials with speeds between 0.8 and $1.2 \mathrm{~m} / \mathrm{s}$, allowing us to match the speeds observed in the participants with stroke.

The participants with stroke were classified based on their DGI score, as measured by a licensed physical therapist. Participants with a DGI score of $>19$ were classified as low fall risk, while participants with a lower DGI score were classified as higher fall risk [20]. Table 1 lists characteristics of the control group, low fall risk stroke group, and higher fall risk stroke group. Participant age did not vary significantly across the three experimental groups (analysis of variance [ANOVA]: $p=0.34$ ). The amount of time since experiencing a stroke was not significantly

Table 1.

Group characteristics of three experimental groups.

\begin{tabular}{lccc}
\hline Characteristic & Control Group & $\begin{array}{c}\text { Low Fall Risk } \\
\text { Hemiparetic Group }\end{array}$ & $\begin{array}{c}\text { Higher Fall Risk } \\
\text { Hemiparetic Group }\end{array}$ \\
\hline No. of Participants & 19 & 9 & 7 \\
Sex (female/male) & $13 / 6$ & $2 / 7$ & $1 / 6$ \\
Paretic Side (right/left) & NA & $6 / 3$ & $4 / 3$ \\
Age, yr (mean \pm SD) & $62 \pm 8$ & $66 \pm 7$ & $59 \pm 13$ \\
$\begin{array}{l}\text { Time Poststroke, mo (median, } \\
\text { range) }\end{array}$ & NA & $29(19-116)$ & $26(9-201)$ \\
$\begin{array}{l}\text { Dynamic Gait Index score } \\
\text { (median, range) }\end{array}$ & NA & $18(9-19)$ & $23(21-24)$ \\
\hline
\end{tabular}

Note: Normally distributed age data are reported as mean \pm SD, while non-normally distributed time poststroke data are reported in terms of median and range. $\mathrm{NA}=$ not applicable, $\mathrm{SD}=$ standard deviation 
different between the low fall risk and higher fall risk groups (Wilcoxon rank sum test: $p=0.26$ ).

\section{Data Collection and Processing}

Spatiotemporal gait characteristics were measured at a sampling rate of $100 \mathrm{~Hz}$ using reflective markers (Vicon; Denver, Colorado) placed on the sacrum and on the left and right heels. The sacrum marker was used as an estimate of mediolateral CoM position. This simple estimate does not consider motion of the limbs, but the timing of pelvis and CoM movements along a mediolateral axis is very similar [25]. For brevity, we will refer to this midline location as the mediolateral position of the CoM. For each step, mediolateral initial swing foot displacement was quantified with respect to the CoM, and mediolateral CoM displacement was quantified with respect to the stance foot (Figure 1). Marker velocities were calculated by low-pass filtering the position data at $20 \mathrm{~Hz}$ and then differentiating with respect to time. Anteroposterior velocities of the heel markers were used to identify heel-strike and toe-off events and thus to

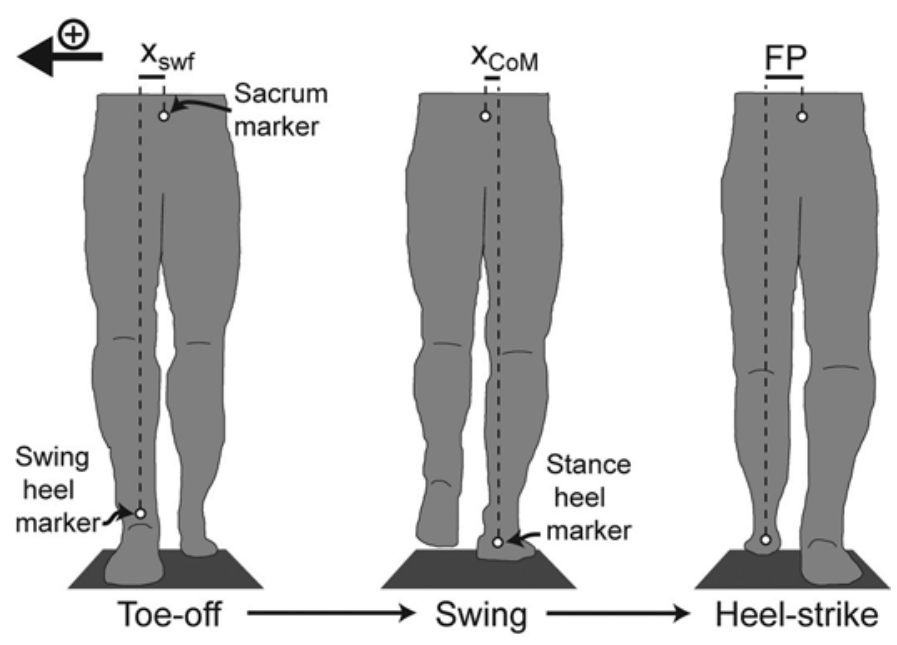

Figure 1.

Mediolateral kinematic measures were quantified at various points in the gait cycle, as illustrated for a single step with the left lower limb (frontal plane view from the posterior side). At toe-off, initial swing foot displacement $\left(\mathrm{x}_{\text {swf }}\right)$ was measured as the mediolateral distance from the center of mass (CoM) to the swing heel. During the first half of swing, mediolateral CoM displacement $\left(\mathrm{x}_{\mathrm{CoM}}\right)$ was quantified relative to the stance heel. Upon heel strike, mediolateral foot placement (FP) was measured as the distance from the CoM to the swing heel. For all steps, the direction toward the swing limb was considered positive. determine stance and swing phases of the gait cycle [26]. For use in subsequent regressions (described later), we calculated the mediolateral position and velocity of the swing foot relative to the CoM at the start of each step (initial swing foot displacement and velocity), the average mediolateral position and velocity of the CoM relative to the stance foot during the first half of the swing phase (CoM displacement and velocity), and the mediolateral location of the swing foot relative to the CoM at the end of the step (defined as mediolateral FP). Note that the magnitude of the initial swing foot velocity was largely determined by the CoM velocity because the mediolateral velocity of the foot is low at this point in the gait cycle.

Bilateral gluteus medius (GM) activity was measured using surface electromyography (EMG) electrodes (Konigsberg Electronics; Monrovia, California) sampled at $1,000 \mathrm{~Hz}$. These EMG data were processed by bandpass filtering between 20 and $500 \mathrm{~Hz}$, rectifying, and low-pass filtering at $50 \mathrm{~Hz}$. EMG data were divided into strides, and the average EMG trace during a stride was calculated for each trial. EMG data were then normalized by the peak value during the average stride, which occurred during the stance phase (typically within the first $25 \%$ of a gait cycle). For use in our regressions, we calculated the average swing limb GM activity during the first half of swing and the average contralateral stance limb GM activity during the same time period.

\section{Data Analysis and Statistics}

Multiple ANOVAs were first performed to determine whether the three experimental groups (control group, low fall risk group, and higher fall risk group) differed in terms of self-selected overground walking speed, analyzed treadmill walking speed, and mediolateral FP location. Where appropriate, post hoc tests were performed (Tukey honestly significant difference). We interpreted $p$ values of less than 0.05 as significant.

To investigate the relationships between gait mechanics and the active control of FP, we performed a series of multiple linear regressions. Our first goal was to identify the factors that influence swing limb GM activity. For this analysis, the dependent variable in the regression was the average activity of the GM during the first half of the swing phase. The independent variables were the average mediolateral CoM displacement, average CoM velocity, and average contralateral stance limb GM activity during this same time period. We performed a 
multiple linear regression for each experimental group in order to identify significant associations. Our second goal was to identify the factors that influence mediolateral FP. For this analysis, the dependent variable in the regression was the mediolateral location of the swing foot with respect to the CoM at the time of heel-strike. The independent variables were the average GM activity during the first half of swing and the initial swing foot displacement and velocity relative to the CoM at the start of the swing phase. Again, a multiple linear regression was used to identify significant associations for each experimental group.

These regression methods were identical to those used in our previous study investigating mediolateral gait stabilization in young, uninjured individuals, with the exception of not including acceleration terms [21]. Specifically, we did not test for effects of mediolateral CoM acceleration on swing limb GM activity or for effects of initial swing foot acceleration on eventual mediolateral FP location. Our reason for not including these acceleration factors is that many participants exhibited a clear relationship between mediolateral displacement and acceleration. Due to the relatively small number of data points included in each regression (as few as 481 in comparison to the 5,000 included in our previous regressions), such collinearity can make the interpretation of regression results more difficult. Importantly, adding these acceleration factors back into the regressions did not influence the presence of significant associations with any of the other independent variables.

For control participants, data from both lower limbs were combined in the regressions because no bilateral differences were observed. For participants with stroke, data from the nonparetic and paretic limbs were analyzed separately. In order to focus on step-to-step differences in behavior rather than differences between individuals, a "dummy" indicator variable was included in each of the regressions to represent limb number. The data distribution within each trial was normalized by subtracting the mean and dividing by the standard deviation. This allowed clearer interpretation of the regression results because a larger regression coefficient would indicate a stronger relationship [27]. For each regression, the maximum variance inflation factor was less than 1.5 (mean = 1.06), well below the value of 10 often taken as a sign of problematic multicollinearity [28]. For all regressions, $p$ values of less than 0.05 were interpreted as significant. For both the low fall risk and higher fall risk groups, cases in which the significance of the regression coefficient differed from the control group were further investigated. Specifically, we determined whether the difference between the coefficients fell outside of the 95 percent confidence interval calculated from the standard error values [29]. This test revealed whether the two regression coefficients should be considered to be significantly different.

\section{RESULTS}

In control participants, the state of the stance limb influenced swing limb GM activity in the contralateral limb, which in turn influenced mediolateral FP. In hemiparetic participants with a low fall risk (DGI >19), the same neuromechanical stabilization strategy was largely retained. However, this strategy was clearly disrupted in hemiparetic participants with a higher fall risk (DGI $\leq 19$ ), particularly for steps taken with the paretic limb.

\section{Group Characteristics}

Table 2 describes gait characteristics for the experimental groups. The control group had a significantly faster self-selected overground walking speed than either of the hemiparetic groups. Importantly, however, the walking speeds during the analyzed treadmill trials did not differ significantly between the three groups. Participants in the higher fall risk hemiparetic group placed their paretic limb significantly more laterally than they placed their nonparetic limb and significantly more laterally than the FP exhibited by the other experimental groups.

\section{Swing Phase Gluteus Medius Activity}

In the control group, swing limb GM activity was associated with the simultaneous state of the contralateral stance limb (Figure 2(a)). Swing limb GM activity was most strongly associated with mediolateral CoM displacement $(p<0.001)$, with higher GM activity during steps in which the CoM was farther from the contralateral stance foot. Swing limb GM activity was also positively associated with CoM velocity ( $p=0.03$ ), with greater activity when the CoM was moving more slowly toward the stance foot. Finally, swing limb GM activity was positively associated with simultaneous GM activity in the contralateral stance limb ( $p<0.001)$, with increased swing phase activity during steps with higher contralateral stance phase activity. 
JRRD, Volume 52, Number 5, 2015

Table 2.

Basic gait behavior measures for three experimental groups.

\begin{tabular}{|c|c|c|c|c|}
\hline Gait Measure & Control Group & $\begin{array}{c}\text { Low Fall Risk } \\
\text { Hemiparetic Group }\end{array}$ & $\begin{array}{c}\text { Higher Fall Risk } \\
\text { Hemiparetic Group }\end{array}$ & $p$-Value \\
\hline $\begin{array}{l}\text { Self-Selected Overground Walk- } \\
\text { ing Speed }(\mathrm{m} / \mathrm{s})\end{array}$ & $1.31 \pm 0.20^{*}$ & $0.96 \pm 0.11$ & $0.79 \pm 0.26$ & $<0.001$ \\
\hline $\begin{array}{l}\text { Analyzed Treadmill Walking } \\
\text { Speed }(\mathrm{m} / \mathrm{s})\end{array}$ & $1.06 \pm 0.14$ & $0.98 \pm 0.24$ & $1.03 \pm 0.26$ & 0.21 \\
\hline $\begin{array}{l}\text { Mediolateral Foot Placement } \\
\text { (\%LL) }\end{array}$ & $11.3 \pm 2.0$ & - & - & $<0.001$ \\
\hline Both Limbs & $11.3 \pm 2.0$ & - & - & - \\
\hline Nonparetic Limb & - & $9.7 \pm 1.9$ & $11.0 \pm 2.3$ & - \\
\hline Paretic Limb & - & $11.8 \pm 2.7$ & $16.9 \pm 4.1^{*}$ & - \\
\hline Analyzed Steps $(n)$ & 3,037 & - & - & NA \\
\hline Nonparetic Limb & - & 707 & 481 & - \\
\hline Paretic Limb & - & 705 & 481 & - \\
\hline \multicolumn{5}{|c|}{$\begin{array}{l}\text { Note: Data are reported as mean } \pm \text { standard deviation where appropriate. For control group, } 57 \text { total walking trials were performed between } 0.8 \text { and } 1.2 \mathrm{~m} / \mathrm{s} \text {, meet- } \\
\text { ing our walking speed requirement, and were included in analysis. For low fall risk group, } 16 \text { self-selected speed trials and } 13 \text { fastest-comfortable speed trials met } \\
\text { walking speed requirement. For higher fall risk group, } 13 \text { self-selected speed trials and } 9 \text { fastest-comfortable speed trials met this requirement. Where reported, } p \text { - } \\
\text { values were calculated using analysis of variance. } \\
\text { *Indicates value significantly larger than all other comparison groups ( }(p<0.05 \text {, Tukey post hoc test). } \\
\% \text { LL = percentage of lower-limb length, NA = not applicable. }\end{array}$} \\
\hline
\end{tabular}

The factors that influence swing limb GM activity in the low fall risk hemiparetic group were largely similar to those in control participants (Figure 2(b)). For steps taken with the nonparetic limb, swing limb GM activity was positively associated with mediolateral CoM displacement $(p<0.001)$, mediolateral CoM velocity ( $p=$ 0.03 ), and simultaneous stance limb GM activity in the contralateral limb $(p=0.009)$. For steps taken with the paretic limb, swing limb GM activity was positively associated with CoM displacement $(p<0.001)$ and stance limb GM activity in the contralateral limb $(p<$ 0.001).

In contrast, the factors that influence swing limb GM activity in the higher fall risk hemiparetic group differed from those in control participants (Figure 2(c)). For nonparetic steps, only mediolateral CoM displacement was significantly ( $p=0.001)$ associated with swing limb GM activity. The differences from control behavior were more apparent for steps taken with the paretic limb. Mediolateral CoM displacement was not significantly associated with swing limb GM activity, with a regression coefficient significantly smaller than for controls. Mediolateral CoM velocity was negatively correlated $(p=$ 0.03 ) with swing limb GM activity, indicating greater GM activity during steps in which the CoM was moving more quickly toward the stance foot. This negative correlation was significantly different than the positive corre- lation present in the control group. A trend for a positive association between swing limb GM activity and contralateral stance limb GM activity nearly achieved the level of significance $(p=0.051)$.

\section{Mediolateral Foot Placement}

In the control group, mediolateral FP was influenced by a combination of swing limb GM activity and the initial mechanical state of the swing limb (Figure 3(a)). FP was positively associated with swing limb GM activity ( $p<$ 0.001 ), with increased hip abductor activity preceding more lateral FP. FP was negatively associated with the mediolateral initial swing foot displacement relative to the CoM $(p<0.001)$, with the foot placed more medially for steps in which it began the swing phase farther from the CoM. Similarly, FP was negatively associated with the initial mediolateral velocity of the swing foot relative to the CoM ( $p<0.001)$, with the foot placed more medially for steps in which the CoM was initially moving more quickly toward the contralateral stance limb.

In the low fall risk hemiparetic group, the factors that influence FP location varied between the nonparetic and paretic limbs (Figure 3(b)). For steps taken with the nonparetic limb, FP was associated with swing limb GM activity $(p=0.001)$, initial swing foot displacement $(p=$ $0.04)$, and initial swing foot velocity $(p=0.002)$, just as in the control group. For paretic steps, FP was only 

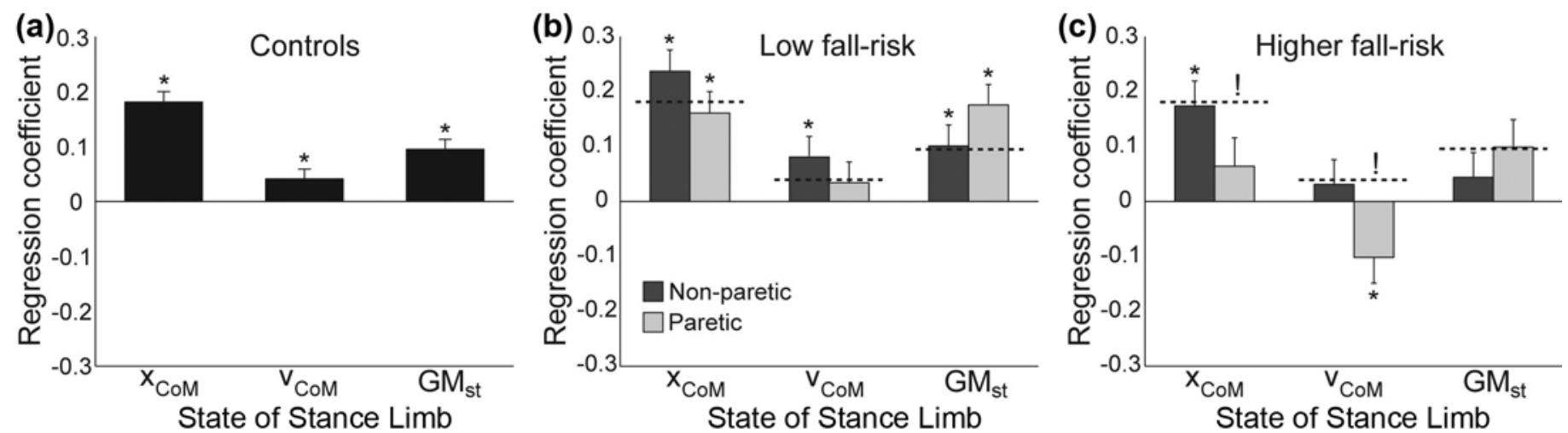

Figure 2.

Swing phase gluteus medius activity was significantly associated with several metrics quantifying the state of the stance limb. The regression coefficients are illustrated for (a) the control group, (b) the low fall risk hemiparetic group, and (c) the higher fall risk hemiparetic group. For all panels, the bar represents the regression coefficient, the error bar represents standard error, and asterisks (*) indicate a significant $(p<0.05)$ association. For purposes of visual comparison, the control group regression coefficients are indicated in panels (b) and (c) using horizontal dashed lines. Exclamation marks (!) indicate a significant difference between the indicated regression coefficient and the matching regression coefficient in the control group. $\mathrm{x}_{\mathrm{CoM}}=\mathrm{center}_{\text {of }}$ mass displacement, $\mathrm{v}_{\mathrm{CoM}}=$ center of mass velocity, $\mathrm{GM}_{\mathrm{st}}=$ stance limb gluteus medius activity.
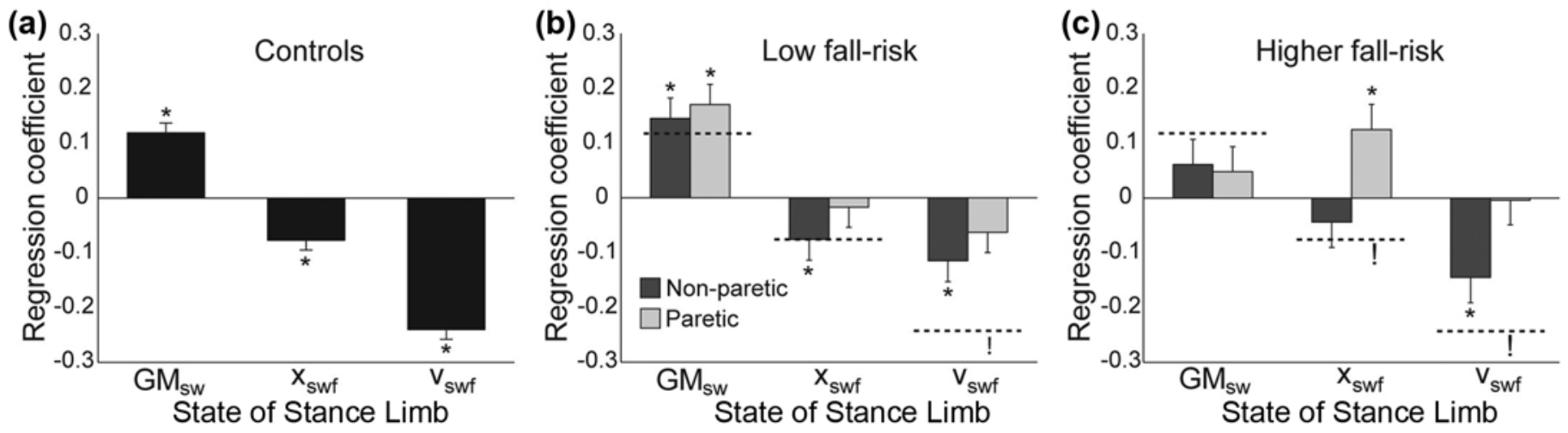

Figure 3.

Mediolateral foot placement was significantly associated with various combinations of swing phase gluteus medius activity (GM $\mathrm{sw}_{\text {) }}$ and metrics quantifying the initial mechanical state of the swing limb. The regression coefficients are illustrated for (a) the control group, (b) the low fall risk hemiparetic group, and (c) the higher fall risk hemiparetic group. For all panels, the bar represents the regression coefficient, the error bar represents standard error, and asterisks ( $*)$ indicate a significant $(p<0.05)$ association. For comparison purposes, the control group regression coefficients are indicated in panels (b) and (c) using horizontal dashed lines. Exclamation marks (!) indicate a significant difference between the indicated regression coefficient and the matching regression coefficient in the control group. $\mathrm{x}_{\text {swf }}=$ initial swing foot displacement, $\mathrm{v}_{\text {swf }}=$ initial swing foot velocity.

significantly associated with swing limb GM activity $(p<$ 0.001 ). The magnitude of the regression coefficient for the initial foot velocity was significantly smaller in this group compared with control participants.

The factors that influence mediolateral FP in the higher fall risk hemiparetic group differed substantially from those in the control group (Figure 3(c)). For nonparetic steps, only the initial foot velocity was significantly ( $p=0.002)$ associated with the eventual FP location. For paretic steps, only the initial foot displacement was significantly $(p=0.006)$ associated with FP. The positive association between initial displacement and final FP 
indicates that the foot was placed more laterally for steps in which it began the swing phase farther from the CoM, significantly different from the negative association observed in control participants. Additionally, the lack of an association between swing foot velocity and FP location differed significantly from controls.

Typical relationships between mediolateral gait motion and paretic limb swing limb GM activity are illustrated for representative participants from the low fall risk and higher fall risk groups (Figure 4). For participants categorized as low fall risk, steps in which the CoM was farther from the stance foot (increased CoM displacement) typically also exhibited clear bursts of paretic swing limb GM activity during swing and more lateral subsequent FP (Figure 4(a)). This mechanicsdependent use of GM activity to modulate FP appeared to be disrupted in the higher fall risk group. Rather than exhibiting a single burst of GM activity early in swing, participants in this group often activated the GM at less predictable points in the gait cycle (Figure 4(b)). These bursts tended to be present when the CoM moved more quickly toward the stance foot and did not consistently influence the subsequent mediolateral FP.

\section{DISCUSSION}

The mechanical demands of bipedal gait require humans to actively ensure their stability. We have previously proposed that neurologically intact humans stabilize their walking patterns by sensing the mechanical state of their stance lower limb and use this information to drive active muscular control of their mediolateral FP [21]. In the current study, this neuromechanical stabilization strategy was found to be present among uninjured older adults. As hypothesized, however, this strategy was disrupted in participants with stroke classified as having an increased fall risk.

The strategy for active FP observed in the control group (of similar age as the participants with stroke) was qualitatively similar to that previously reported for young, uninjured participants [21]. Swing limb GM activity

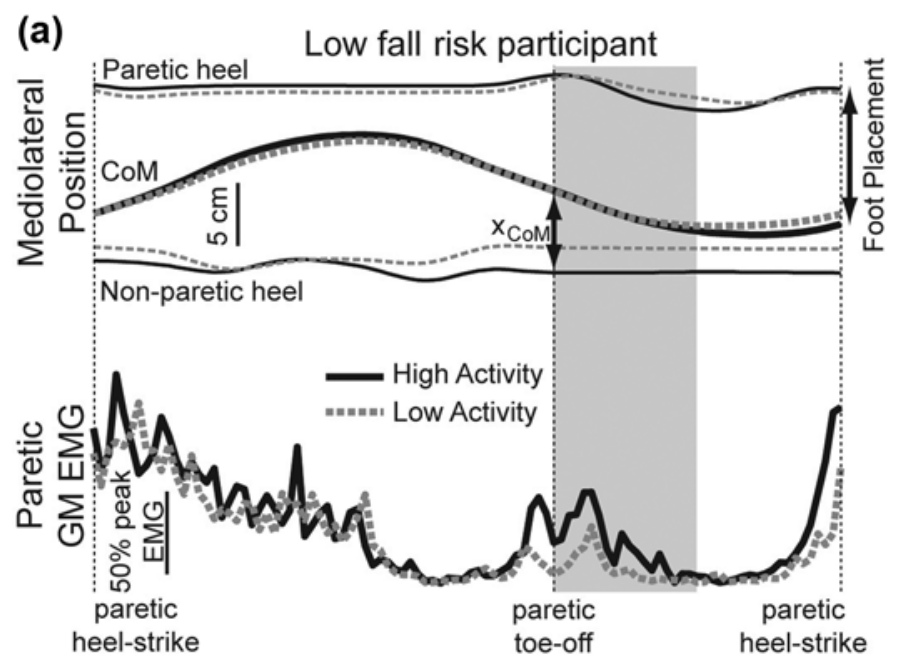

Stride

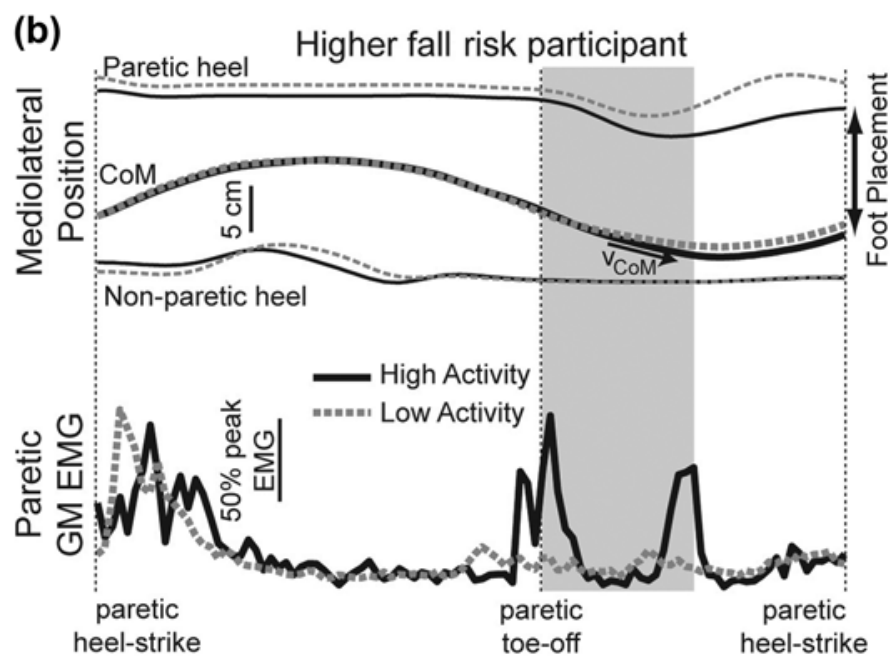

Stride

Figure 4.

Mediolateral marker positions and paretic limb gluteus medius (GM) activity over the course of a stride are illustrated for typical walking trials. Each line represents the average of five strides (from paretic heel-strike to next paretic heel-strike). High-activity strides were those with the greatest paretic limb GM activity during the first half of swing (shaded area), and low-activity strides were those with the lowest paretic limb GM activity during this period. (a) For the low fall risk participant, the center of mass (CoM) displacement $\left(\mathrm{x}_{\mathrm{CoM}}\right)$ from the nonparetic stance foot was larger during high-activity strides, which ended with the paretic limb being placed more laterally (increased foot placement distance). (b) For the higher fall risk participant, the CoM moved more quickly toward the stance foot (indicated by steeper slope of CoM velocity $\left[\mathrm{v}_{\mathrm{CoM}}\right]$ ) for the first half of swing during high-activity strides. The presence of increased paretic limb GM activity did not cause the paretic limb to be placed more laterally. EMG = electromyography. 
was most strongly associated with the mediolateral position of the CoM with respect to the contralateral stance foot and was more weakly associated with CoM velocity and contralateral stance limb GM activity. This result supports the proposal that older adults actively stabilize their gait based on their perception of CoM mechanics. The magnitude of the swing limb GM activity was associated with the subsequent mediolateral FP at the end of the step, indicating the presence of useful active control. As in the young [21], final FP location was also associated with the initial displacement and velocity of this foot relative to the CoM at the start of the step. This finding can likely be attributed to passive pendular behavior of the swing limb, with the leg's initial mechanical state likely influencing the mediolateral trajectory during swing $[21,30]$. The observed mechanism of actively controlling FP based on the mechanical state of the stance limb supports the predictions of previous model simulations [31]. Our results also provide evidence for a neuromechanical mechanism that humans may use to accomplish the apparent goal of maintaining a certain margin of stability between their extrapolated CoM (a metric combining CoM position and CoM velocity) and their FP [32-33].

Among participants with stroke classified as low fall risk (DGI >19), the FP strategy was largely similar to that in control participants. For steps taken with both the nonparetic and paretic limbs, swing limb GM activity was greater when the CoM was farther from the stance foot and the stance limb GM activity was more active. Increased swing limb GM activity was followed by more lateral FP, indicating that the mechanism of actively controlling FP location is essentially retained within this group. However, the influence of the swing limb's initial mechanical state on its subsequent FP location was reduced for steps taken with the paretic limb. This apparent reduction in the importance of passive pendular mechanics may reflect an inability to allow the paretic limb to swing freely while stepping forward, possibly due to abnormal joint torques attributable to dyscoordination in muscle activation [34].

While the control and low fall risk groups appeared to base their swing limb GM activity on similar stance limb characteristics, this strategy was disrupted in the higher fall risk group (DGI $\leq 19$ ). For steps taken with the nonparetic limb, swing limb GM activity was most strongly influenced by the CoM displacement from the stance foot, as in control participants. However, this rela- tionship between CoM mechanics and swing limb control was altered for steps with the paretic limb. The CoM displacement from the stance foot did not influence GM activity, with a significantly weaker association than in control participants. The lack of a clear effect of CoM displacement may be explained by poststroke disruptions in sensory accuracy [35], sensorimotor integration [36], or precise muscular activation [37]. An inability to either develop an accurate perception of CoM and swing limb location or appropriately modulate swing limb GM activity could prevent the use of the typical stabilization strategy. Additionally, swing limb GM activity was greater during steps in which the CoM was moving more quickly toward the stance foot, opposite to the relationship in controls. Speculatively, this response may be attributed to a balance reaction in which the swing limb is used to control frontal plane angular momentum [38]. Such a balance reaction is reminiscent of behavior during unipedal standing, in which balance can be maintained by repositioning the limb not in contact with the ground [38-39], as evidenced by bursts of GM activity in this limb immediately following pushes of the CoM toward the stance limb [40]. People with stroke are often overly reliant on their nonparetic limb musculature for stability [41] and walk with their CoM closer mediolaterally to their nonparetic limb than their paretic limb [42]. The present results are consistent with the higher fall risk group attempting to maintain their gait stability by balancing their CoM primarily over the nonparetic limb while placing their paretic limb quite laterally (Table 2) rather than following the typical strategy of controlling FP location to accelerate the CoM toward the midline with each step [33].

In addition to altered activation of the GM, the factors that influence mediolateral FP location also differed in the higher fall risk group. Unlike in control participants and the low fall risk group, swing limb GM activity was not significantly associated with FP location. The reduced ability of GM activity to cause more lateral FP may be due to the decreased hip abduction strength of many patients with stroke [43] or an inability to appropriately produce independent GM contractions and isolated frontal plane hip torques [43-45]. Within the higher fall risk group, the initial mechanical state of the paretic swing limb also had an altered relationship with its eventual FP location. Specifically, the paretic limb's FP was positively related to its position at the start of the step; if the foot began the step relatively laterally, it also ended with the step being placed relatively laterally. Rather than FP 
being determined by a combination of passive lower-limb mechanics and active control [21], it seems that higher fall risk individuals approximately maintained the relative mediolateral position of the paretic limb across the course of a step. This finding may indicate that these individuals were unable to appropriately vary their FP location in response to step-to-step changes in the mechanical demands of the task, thereby reducing the "good variance" thought to typically contribute to mediolateral gait stabilization [46-47]. Instead, people with stroke with a reduced ability to accurately position their paretic limb may simply target a FP location that successfully prevented a fall during the previous paretic step.

The present results are an early step toward a mechanistic understanding of gait instability poststroke. Such an understanding could allow for the development of more effective approaches to stroke rehabilitation. Based on previous [21] and current results, the typical neuromechanical stabilization strategy during gait appears to involve the perception of CoM mechanics. To determine how this strategy may be disrupted following a stroke, it will be necessary to identify the sources of sensory information used to form this perception, possibly including proprioceptive feedback from the hip, knee, or ankle [4850]; feedback of cutaneous pressure from the stance foot [51]; visual feedback [52]; or vestibular feedback [53]. An understanding of the relevant sensory sources could motivate the introduction of new therapy methods, such as training to improve proprioception [54]. A second component of the typical stabilization strategy is the modulation of mediolateral FP through active control of the swing limb. While in its early stages, gait training that includes practice stepping to targets may improve mobility by allowing more accurate FP [55].

An understanding of the mechanism underlying decreased gait stability following a stroke is particularly important given the recent failure of several intervention methods to reduce fall risk. In randomized controlled trials, the rate of falls among people with stroke was not preferentially reduced by either a multifactorial falls prevention program [56] or a task-related balance and strengthening program [57]. These results were surprising given the previous success of similar interventions in improving balance among uninjured older adults [5859]. It is possible that the cause of increased fall risk differs between these two populations [3]. Indeed, the present results suggest that a neuromechanical strategy for mediolateral stabilization initially suggested in a young population was essentially retained in an older control group but was disrupted among people with stroke thought to be at an increased risk for falls [21]. Additionally, an understanding of the factors contributing to gait instability may not directly follow from investigations of quiet standing posture. From a basic mechanical perspective, mediolateral stabilization strategies that are effective during standing posture (e.g., resisting perturbations solely by increasing nonparetic limb abduction or adduction torques) would not be effective during gait, in which the paretic limb is forced to play a role [41].

Due to our focus on walking stability, participants were classified using their DGI score, a metric intended to quantify various aspects of balance during gait [11]. While DGI score has been related to self-reported falls among older adults, the sensitivity (59\%) and specificity (64\%) values were only moderate [20]. Among people with stroke, the DGI has been validated with respect to other measures of balance (e.g., BBS, ABC) but has not yet been shown to predict fall risk [12]. While the recently introduced modified DGI appears to have improved psychometric properties, this metric should still not be considered a gold standard for quantifying poststroke gait stability [60]. Despite the limitations of our classifications based on DGI score, we observed clear differences in walking behavior between the low fall risk and higher fall risk groups. Future work based on the present results may allow us to better understand the causes of gait instability that are currently only hinted at by clinical measures such as the DGI.

Our ability to extrapolate the present results to a more general understanding of poststroke falls is limited by several characteristics of the experiments. We restricted our analysis to relatively fast walking speeds ( $\geq 0.8 \mathrm{~m} / \mathrm{s}$ ) so the observed changes in gait stabilization strategy may not be relevant for individuals who walk more slowly. However, even people with stroke with an ability to walk faster than $0.8 \mathrm{~m} / \mathrm{s}$ have a higher incidence of falls than uninjured older adults [57,61]. In the present analysis, we focused on a FP strategy during unperturbed walking. While our approach will not provide insight into the response to mechanical perturbations like trips or slips, many poststroke falls are due to "internal perturbations" that can be caused by the type of inaccurate control investigated here [3]. Finally, the muscle activity of the hip adductors was not quantified in the present study. Swing phase activity of the adductors may contribute to mediolateral FP, although the effects of adductor magnus 
activity in young controls were much smaller than the effects of GM activity [21].

Our use of regressions to quantify relationships between measures of gait kinematics and muscle activity is also a limitation. Most importantly, the reported significant associations cannot provide insight into causality. For example, the positive association between CoM displacement and swing limb GM activity in control participants does not necessarily mean that increased displacements cause increased muscle activity. However, our previous experiments that used perturbations to alter CoM displacement and subsequently quantified GM activity have provided stronger evidence for such a causal relationship [21]. The variable number of steps included in each regression (ranging from 481 to 3,037 steps) affects the probability that each regression would identify significant associations. A reduced number of steps included in the regression for the higher fall risk group likely explains why some associations reported as significant among controls did not reach significance in this group (see " $\mathrm{GM}_{\mathrm{st}}$ " in Figure 2(c)). Despite this limitation, several clear, statistically significant differences between gait behaviors were identified between groups.

\section{CONCLUSIONS}

Poststroke participants classified as having a higher fall risk exhibited an altered gait stabilization strategy, particularly for steps taken with the paretic lower limb. This subgroup of participants with stroke did not follow the typical strategy of actively controlling their mediolateral FP in response to step-by-step variation in CoM mechanics. The present results have the potential to serve as an early step in identifying mechanistic causes of limited gait stability poststroke and could lead to novel rehabilitation strategies.

\section{ACKNOWLEDGMENTS}

\author{
Author Contributions: \\ Study concept and design: J. C. Dean, S. A. Kautz. \\ Acquisition of data: S. A. Kautz. \\ Analysis and interpretation of data: J. C. Dean, S. A. Kautz. \\ Processing of data: J. C. Dean. \\ Drafting of manuscript: J. C. Dean, S. A. Kautz. \\ Study supervision: S. A. Kautz.
}

Financial Disclosures: The authors have declared that no competing interests exist.
Funding/Support: This material was based on work supported by the Department of Veterans Affairs (VA) Rehabilitation Research and Development Service (grant 1 IK2 RX000750) and the National Institutes of Health (grants HD46820 and GM109040).

Institutional Review: Recruitment and informed consent procedures were approved by the University of Florida Institutional Review Board (protocol 46-2004).

Participant Follow-Up: The authors do not plan to inform participants of the publication of this study. Participants initially signed an informed consent form stating that the results of this study would be published.

Disclaimer: The views expressed by the authors are their own and do not necessarily reflect the official policy of the VA or the U.S. Government.

\section{REFERENCES}

1. Weerdesteyn V, de Niet M, van Duijnhoven HJ, Geurts AC. Falls in individuals with stroke. J Rehabil Res Dev. 2008; 45(8):1195-1213. [PMID:19235120] http://dx.doi.org/10.1682/JRRD.2007.09.0145

2. Hyndman D, Ashburn A, Stack E. Fall events among people with stroke living in the community: Circumstances of falls and characteristics of fallers. Arch Phys Med Rehabil. 2002;83(2):165-70. [PMID:11833018] http://dx.doi.org/10.1053/apmr.2002.28030

3. Jørgensen L, Engstad T, Jacobsen BK. Higher incidence of falls in long-term stroke survivors than in population controls: Depressive symptoms predict falls after stroke. Stroke. 2002;33(2):542-47. [PMID:11823667] http://dx.doi.org/10.1161/hs0202.102375

4. Harris JE, Eng JJ, Marigold DS, Tokuno CD, Louis CL. Relationship of balance and mobility to fall incidence in people with chronic stroke. Phys Ther. 2005;85(2):150-58. [PMID:15679466]

5. Soyuer F, Oztürk A. The effect of spasticity, sense and walking aids in falls of people after chronic stroke. Disabil Rehabil. 2007;29(9):679-87. [PMID:17453990] http://dx.doi.org/10.1080/09638280600925860

6. Schmid AA, Van Puymbroeck M, Altenburger PA, Dierks TA, Miller KK, Damush TM, Williams LS. Balance and balance self-efficacy are associated with activity and participation after stroke: A cross-sectional study in people with chronic stroke. Arch Phys Med Rehabil. 2012;93(6): 1101-7. [PMID:22502804] http://dx.doi.org/10.1016/j.apmr.2012.01.020

7. Hellström K, Lindmark B, Wahlberg B, Fugl-Meyer AR. Self-efficacy in relation to impairments and activities of daily living disability in elderly patients with stroke: A prospective investigation. J Rehabil Med. 2003;35(5):202-7.

[PMID:14582550] http://dx.doi.org/10.1080/16501970310000836 
8. Blum L, Korner-Bitensky N. Usefulness of the Berg Balance Scale in stroke rehabilitation: A systematic review. Phys Ther. 2008;88(5):559-66. [PMID:18292215] http://dx.doi.org/10.2522/ptj.20070205

9. Maeda N, Kato J, Shimada T. Predicting the probability for fall incidence in stroke patients using the Berg Balance Scale. J Int Med Res. 2009;37(3):697-704.

[PMID:19589253] http://dx.doi.org/10.1177/147323000903700313

10. Tilson JK, Wu SS, Cen SY, Feng Q, Rose DR, Behrman AL, Azen SP, Duncan PW. Characterizing and identifying risk for falls in the LEAPS study: A randomized clinical trial of interventions to improve walking poststroke. Stroke. 2012;43(2):446-52. [PMID:22246687] http://dx.doi.org/10.1161/STROKEAHA.111.636258

11. Shumway-Cook A, Woollacott MH. Motor control: Translating research into clinical practice. 4th ed. Philadelphia (PA): Wolters Kluwer Health, Lippincott Williams \& Wilkins; 2012.

12. Jonsdottir J, Cattaneo D. Reliability and validity of the dynamic gait index in persons with chronic stroke. Arch Phys Med Rehabil. 2007;88(11):1410-15.

[PMID:17964880]

http://dx.doi.org/10.1016/j.apmr.2007.08.109

13. Ng SS. Contribution of subjective balance confidence on functional mobility in subjects with chronic stroke. Disabil Rehabil. 2011;33(23-24):2291-98. [PMID:21473685] http://dx.doi.org/10.3109/09638288.2011.568667

14. Hausdorff JM, Rios DA, Edelberg HK. Gait variability and fall risk in community-living older adults: A 1-year prospective study. Arch Phys Med Rehabil. 2001;82(8):105056. [PMID:11494184]

http://dx.doi.org/10.1053/apmr.2001.24893

15. Balasubramanian CK, Neptune RR, Kautz SA. Variability in spatiotemporal step characteristics and its relationship to walking performance post-stroke. Gait Posture. 2009; 29(3):408-14. [PMID:19056272] http://dx.doi.org/10.1016/j.gaitpost.2008.10.061

16. Nott CR, Neptune RR, Kautz SA. Relationships between frontal-plane angular momentum and clinical balance measures during post-stroke hemiparetic walking. Gait Posture. 2014;39(1):129-34. [PMID:23820449] http://dx.doi.org/10.1016/j.gaitpost.2013.06.008

17. Herman T, Giladi N, Gurevich T, Hausdorff JM. Gait instability and fractal dynamics of older adults with a "cautious" gait: Why do certain older adults walk fearfully? Gait Posture. 2005;21(2):178-85. [PMID:15639397] http://dx.doi.org/10.1016/j.gaitpost.2004.01.014

18. Granata KP, Lockhart TE. Dynamic stability differences in fall-prone and healthy adults. J Electromyogr Kinesiol. 2008;18(2):172-78. [PMID:17686633] http://dx.doi.org/10.1016/j.jelekin.2007.06.008
19. Lockhart TE, Liu J. Differentiating fall-prone and healthy adults using local dynamic stability. Ergonomics. 2008; 51(12):1860-72. [PMID:19034782] http://dx.doi.org/10.1080/00140130802567079

20. Shumway-Cook A, Baldwin M, Polissar NL, Gruber W. Predicting the probability for falls in community-dwelling older adults. Phys Ther. 1997;77(8):812-19. [PMID:9256869]

21. Rankin BL, Buffo SK, Dean JC. A neuromechanical strategy for mediolateral foot placement in walking humans. J Neurophysiol. 2014;112(2):374-83. [PMID:24790168] http://dx.doi.org/10.1152/jn.00138.2014

22. Hak L, Houdijk H, Beek PJ, van Dieën JH. Steps to take to enhance gait stability: The effect of stride frequency, stride length, and walking speed on local dynamic stability and margins of stability. PLoS ONE. 2013;8(12):e82842.

[PMID:24349379]

http://dx.doi.org/10.1371/journal.pone.0082842

23. Helbostad JL, Moe-Nilssen R. The effect of gait speed on lateral balance control during walking in healthy elderly. Gait Posture. 2003;18(2):27-36. [PMID:14654205] http://dx.doi.org/10.1016/S0966-6362(02)00197-2

24. Perry J, Garrett M, Gronley JK, Mulroy SJ. Classification of walking handicap in the stroke population. Stroke. 1995;26(6):982-89. [PMID:7762050] http://dx.doi.org/10.1161/01.STR.26.6.982

25. Whittle MW. Three-dimensional motion of the center of gravity of the body during walking. Hum Mov Sci. 1997;16:347-55.

http://dx.doi.org/10.1016/S0167-9457(96)00052-8

26. Zeni JA Jr, Richards JG, Higginson JS. Two simple methods for determining gait events during treadmill and overground walking using kinematic data. Gait Posture. 2008; 27(4):710-14. [PMID:17723303]

http://dx.doi.org/10.1016/j.gaitpost.2007.07.007

27. Schielzeth H. Simple means to improve the interpretability of regression coefficients. Methods Ecol Evol. 2010;1: 103-13. http://dx.doi.org/10.1111/j.2041-210X.2010.00012.x

28. Belsey DA, Kuh H, Welsch RE. Regression diagnostics: Identifying influential data and sources of collinearity. New York (NY): Wiley; 1980.

29. Altman DG, Bland JM. Interaction revisited: The difference between two estimates. BMJ. 2003;326(7382):219.

[PMID:12543843]

http://dx.doi.org/10.1136/bmj.326.7382.219

30. Osaki Y, Kunin M, Cohen B, Raphan T. Three-dimensional kinematics and dynamics of the foot during walking: A model of central control mechanisms. Exp Brain Res. 2007;176(3):476-96. [PMID:16917770] http://dx.doi.org/10.1007/s00221-006-0633-1 
31. Kuo AD. Stabilization of lateral motion in passive dynamic walking. Int J Robot Res. 1999;18:917-30.

http://dx.doi.org/10.1177/02783649922066655

32. Hof AL. The 'extrapolated center of mass' concept suggests a simple control of balance in walking. Hum Mov Sci. 2008;27(1):112-25. [PMID:17935808] http://dx.doi.org/10.1016/j.humov.2007.08.003

33. Hof AL, Vermerris SM, Gjaltema WA. Balance responses to lateral perturbations in human treadmill walking. J Exp Biol. 2010;213(15):2655-64. [PMID:20639427] http://dx.doi.org/10.1242/jeb.042572

34. Clark DJ, Ting LH, Zajac FE, Neptune RR, Kautz SA. Merging of healthy motor modules predicts reduced locomotor performance and muscle coordination complexity post-stroke. J Neurophysiol. 2010;103(2):844-57.

[PMID:20007501]

http://dx.doi.org/10.1152/jn.00825.2009

35. Connell LA, Lincoln NB, Radford KA. Somatosensory impairment after stroke: Frequency of different deficits and their recovery. Clin Rehabil. 2008;22(8):758-67.

[PMID:18678576]

http://dx.doi.org/10.1177/0269215508090674

36. Machado S, Cunha M, Velasques B, Minc D, Teixeira S, Domingues CA, Silva JG, Bastos VH, Budde H, Cagy M, Basile L, Piedade R, Ribeiro P. Sensorimotor integration: Basic concepts, abnormalities related to movement disorders and sensorimotor training-induced cortical reorganization. Rev Neurol. 2010;51(7):427-36. [PMID:20859923]

37. Garland SJ, Gray VL, Knorr S. Muscle activation patterns and postural control following stroke. Motor Control. 2009;13(4):387-411. [PMID:20014647]

38. Hofmann A, Popovic M, Herr H. Exploiting angular momentum to enhance bipedal center-of-mass control. Proceedings of the IEEE International Conference on Robotics and Automation; 2009 May 12-17; Kobe, Japan. p. 4423-29.

39. Federolf P, Roos L, Nigg BM. Analysis of the multisegmental postural movement strategies utilized in bipedal, tandem and one-leg stance as quantified by a principal component decomposition of marker coordinates. J Biomech. 2013;46(15):2626-33. [PMID:24021753] http://dx.doi.org/10.1016/j.jbiomech.2013.08.008

40. Santos MJ, Aruin AS. Effects of lateral perturbations and changing stance conditions on anticipatory postural adjustment. J Electromyogr Kinesiol. 2009;19(3):532-41.

[PMID:18249139]

http://dx.doi.org/10.1016/j.jelekin.2007.12.002

41. Kirker SG, Simpson DS, Jenner JR, Wing AM. Stepping before standing: Hip muscle function in stepping and standing balance after stroke. J Neurol Neurosurg Psychiatry. 2000;68(4):458-64. [PMID:10727481]

http://dx.doi.org/10.1136/jnnp.68.4.458
42. Balasubramanian CK, Neptune RR, Kautz SA. Foot placement in a body reference frame during walking and its relationship to hemiparetic walking performance. Clin Biomech (Bristol, Avon). 2010;25(5):483-90.

[PMID:20193972]

http://dx.doi.org/10.1016/j.clinbiomech.2010.02.003

43. Neckel N, Pelliccio M, Nichols D, Hidler J. Quantification of functional weakness and abnormal synergy patterns in the lower limb of individuals with chronic stroke. J Neuroeng Rehabil. 2006;3:17. [PMID:16857059] http://dx.doi.org/10.1186/1743-0003-3-17

44. Cruz TH, Dhaher YY. Impaired lower limb muscle synergies post-stroke. Conf Proc IEEE Eng Med Biol Soc. 2009;2009:3956-59. [PMID:19964327]

45. Sulzer JS, Gordon KE, Dhaher YY, Peshkin MA, Patton JL. Preswing knee flexion assistance is coupled with hip abduction in people with stiff-knee gait after stroke. Stroke. 2010;41(8):1709-14. [PMID:20576947] http://dx.doi.org/10.1161/STROKEAHA.110.586917

46. Krishnan V, Rosenblatt NJ, Latash ML, Grabiner MD. The effects of age on stabilization of the mediolateral trajectory of the swing foot. Gait Posture. 2013;38(4):923-28.

[PMID:23711985]

http://dx.doi.org/10.1016/j.gaitpost.2013.04.023

47. Rosenblatt NJ, Hurt CP, Latash ML, Grabiner MD. An apparent contradiction: Increasing variability to achieve greater precision? Exp Brain Res. 2014;232(2):403-13.

[PMID:24162866]

http://dx.doi.org/10.1007/s00221-013-3748-1

48. Kavounoudias A, Gilhodes JC, Roll R, Roll JP. From balance regulation to body orientation: Two goals for muscle proprioceptive information processing? Exp Brain Res. 1999;124(1):80-88. [PMID:9928792]

http://dx.doi.org/10.1007/s002210050602

49. Popov KE, Kozhina GV, Smetanin BN, Shlikov VY. Postural responses to combined vestibular and hip proprioceptive stimulation in man. Eur J Neurosci. 1999;11(9):3307-11. [PMID:10510195] http://dx.doi.org/10.1046/j.1460-9568.1999.00733.x

50. Cammarata ML, Dhaher YY. Proprioceptive acuity in the frontal and sagittal planes of the knee: A preliminary study. Eur J Appl Physiol. 2011;111(7):1313-20.

[PMID:21140163] http://dx.doi.org/10.1007/s00421-010-1757-3

51. Kavounoudias A, Roll R, Roll JP. Foot sole and ankle muscle inputs contribute jointly to human erect posture regulation. J Physiol. 2001;532(Pt 3):869-78. [PMID:11313452] http://dx.doi.org/10.1111/j.1469-7793.2001.0869e.x

52. O’Connor SM, Kuo AD. Direction-dependent control of balance during walking and standing. J Neurophysiol. 2009;102(3):1411-19. [PMID:19553493] http://dx.doi.org/10.1152/jn.00131.2009 
53. Bent LR, McFadyen BJ, Inglis JT. Vestibular contributions during human locomotor tasks. Exerc Sport Sci Rev. 2005;33(3):107-13. [PMID:16006817] http://dx.doi.org/10.1097/00003677-200507000-00002

54. Wong JD, Kistemaker DA, Chin A, Gribble PL. Can proprioceptive training improve motor learning? J Neurophysiol. 2012;108(12):3313-21. [PMID:22972960] http://dx.doi.org/10.1152/jn.00122.2012

55. Heeren A, van Ooijen M, Geurts AC, Day BL, Janssen TW, Beek PJ, Roerdink M, Weerdesteyn V. Step by step: A proof of concept study of C-Mill gait adaptability training in the chronic phase after stroke. J Rehabil Med. 2013; 45(7):616-22. [PMID:23811818] http://dx.doi.org/10.2340/16501977-1180

56. Batchelor FA, Hill KD, Mackintosh SF, Said CM, Whitehead $\mathrm{CH}$. Effects of a multifactorial falls prevention program for people with stroke returning home after rehabilitation: A randomized controlled trial. Arch Phys Med Rehabil. 2012;93(9):1648-55. [PMID:22503739] http://dx.doi.org/10.1016/j.apmr.2012.03.031

57. Dean CM, Rissel C, Sherrington C, Sharkey M, Cumming RG, Lord SR, Barker RN, Kirkham C, O’Rourke S. Exercise to enhance mobility and prevent falls after stroke: The community stroke club randomized trial. Neurorehabil Neural Repair. 2012;26(9):1046-57. [PMID:22544817] http://dx.doi.org/10.1177/1545968312441711

58. Sherrington C, Whitney JC, Lord SR, Herbert RD, Cumming RG, Close JC. Effective exercise for the prevention of falls: A systematic review and meta-analysis. J Am Geriatr Soc. 2008;56(12):2234-43. [PMID:19093923] http://dx.doi.org/10.1111/j.1532-5415.2008.02014.x
59. Neyens JC, van Haastregt JC, Dijcks BP, Martens M, van den Heuvel WJ, de Witte LP, Schols JM. Effectiveness and implementation aspects of interventions for preventing falls in elderly people in long-term care facilities: A systematic review of RCTs. J Am Med Dir Assoc. 2011;12(6):410-25. [PMID:21450201] http://dx.doi.org/10.1016/j.jamda.2010.07.018

60. Shumway-Cook A, Taylor CS, Matsuda PN, Studer MT, Whetten BK. Expanding the scoring system for the dynamic gait index. Phys Ther. 2013;93(11):1493-1506. [PMID:23813090] http://dx.doi.org/10.2522/ptj.20130035

61. Rubenstein LZ. Falls in older people: Epidemiology, risk factors and strategies for prevention. Age Ageing. 2006; 35(Suppl 2):ii37-41. [PMID:16926202] http://dx.doi.org/10.1093/ageing/afl084

Submitted for publication September 4, 2014. Accepted in revised form April 14, 2015.

This article and any supplementary material should be cited as follows:

Dean JC, Kautz SA. Foot placement control and gait instability among people with stroke. J Rehabil Res Dev. 2015;52(5):577-90.

http://dx.doi.org/10.1682/JRRD.2014.09.0207

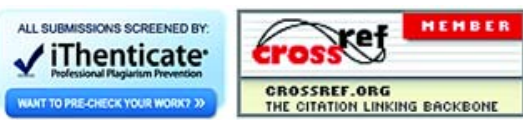

